\title{
The recognition of ubiquitinated proteins by the proteasome
}

\author{
Guinevere L. Grice ${ }^{1} \cdot$ James A. Nathan ${ }^{1}$
}

Received: 23 March 2016/Revised: 22 April 2016/ Accepted: 26 April 2016/Published online: 2 May 2016

(C) The Author(s) 2016. This article is published with open access at Springerlink.com

\begin{abstract}
The ability of ubiquitin to form up to eight different polyubiquitin chain linkages generates complexity within the ubiquitin proteasome system, and accounts for the diverse roles of ubiquitination within the cell. Understanding how each type of ubiquitin linkage is correctly interpreted by ubiquitin binding proteins provides important insights into the link between chain recognition and cellular fate. A major function of ubiquitination is to signal degradation of intracellular proteins by the $26 \mathrm{~S}$ proteasome. Lysine-48 (K48) linked polyubiquitin chains are well established as the canonical signal for proteasomal degradation, but recent studies show a role for other ubiquitin linked chains in facilitating degradation by the $26 \mathrm{~S}$ proteasome. Here, we review how different types of polyubiquitin linkage bind to ubiquitin receptors on the $26 \mathrm{~S}$ proteasome, how they signal degradation and discuss the implications of ubiquitin chain linkage in regulating protein breakdown by the proteasome.
\end{abstract}

Keywords Proteasome - Ubiquitin · Polyubiquitin chains · Ubiquitin binding protein - Ubiquitin binding domain .

Ubiquitin receptors

\section{Introduction}

Diversity in the ubiquitin system is generated by the ability of ubiquitin to form eight different types of chains on itself through its seven lysine (K) residues (K6, K11, K27, K29,

James A. Nathan

jan33@cam.ac.uk

1 Department of Medicine, Cambridge Institute for Medical Research, University of Cambridge, Cambridge CB2 0XY, UK
$\mathrm{K} 33$, K48, and K63) or N-terminus (Met-linked or linear). Further complexity is generated by the formation of polyubiquitin chains of uniform linkage (homotypic) or mixed linkages (heterotypic). Each type of polyubiquitin chain has the potential to act as a distinct intracellular signal that must be specifically identified and decoded by ubiquitin binding proteins (UBPs), to facilitate the diverse outcomes of ubiquitination within cells (reviewed in [1]). This is typically exemplified by the role of K48-polyubiquitin chains signalling proteasomal degradation, while K63-polyubiquitin conjugates are involved in non-proteasomal pathways, including intracellular signalling, DNA repair, and the endosomal-lysosomal system.

Degradation of intracellular soluble proteins is principally mediated by the $26 \mathrm{~S}$ proteasome. This $2.5 \mathrm{MDa}$ complex comprises the cylindrical $20 \mathrm{~S}$ chamber, where proteolysis occurs, and the $19 \mathrm{~S}$ regulatory particle, which binds polyubiquitinated proteins and governs their entry into the 20S catalytic chamber. Through its six ATPase subunits, the 19S complex catalyzes gate opening in the $20 \mathrm{~S}$ outer ring, substrate unfolding, and translocation into the 20S's internal proteolytic chamber. Polyubiquitinated substrates are recognized, tightly bound, and efficiently degraded by the eukaryotic $26 \mathrm{~S}$ through several carefully integrated steps: (1) The initial binding step involves high affinity ubiquitin receptor sites [19S subunits Rpn10 (S5a) and Rpn13] [2-4]. This step is ATP-activated but is easily reversible [2]. (2) Some chains then become more tightly bound and committed to proteolysis, through a process requiring both an unfolded domain within the ubiquitinated protein and ATP hydrolysis [2]. (3) Proteins are deubiquitinated by one or more of the 26S-associated deubiquitinating enzymes (DUBs), Usp14, Uch37, or Rpn11 [5]. (4) Finally, the protein is unfolded and translocated through the opened gate of the 20S [6-8]. 


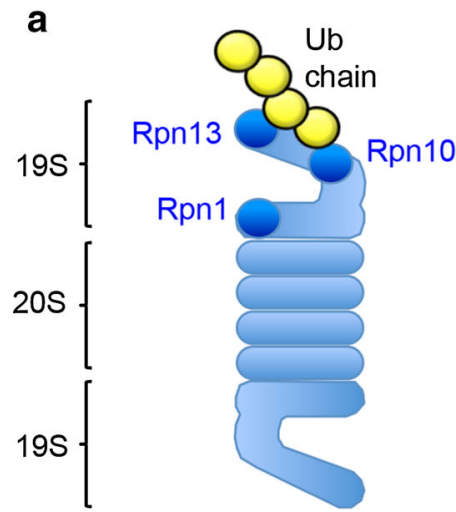

Ub receptors within the 265 proteasome
Fig. 1 Proteasome ubiquitin receptors. Schematic of the 26S proteasome illustrating the position of the intrinsic proteasome receptors (a) and the association of the UBA-Ubl proteins with the $19 \mathrm{~S}$ proteasome (b). Rpn10 and Rpn13 are the predominant high affinity sites for binding ubiquitinated proteins. In yeast, Rpn1 may also

While K48-linked polyubiquitin chains are established as the canonical signal for proteasomal degradation, recent studies highlight the ability of the proteasome to bind monoubiquitinated proteins or other types of polyubiquitin chains. Here, we discuss how ubiquitinated proteins bind to the $26 \mathrm{~S}$ proteasome, and focus on the ability of the proteasome to distinguish between different polyubiquitin linkages.

\section{Proteasome ubiquitin receptors}

The outcome of polyubiquitination is dependent on recognition of the ubiquitin chains by UBPs, which act as ubiquitin receptors. These proteins usually encode several ubiquitinbinding domains (UBDs) that may differ in their affinity and avidity for distinct ubiquitin linkages. UBDs typically consist of one or more $\alpha$-helices that bind ubiquitin through a hydrophobic region, centered on isoleucine-44 (I44). Most UBDs of the $\alpha$-helical class have relatively low affinities for a single ubiquitin molecule, but micromolar affinities for tetraubiquitin chains [9]. The presence of several UBDs within the same protein not only increases the affinity for polyubiquitin chains but can also change the avidity and determines linkage selectivity (reviewed in [10]).

The first ubiquitin receptor identified within the 26S proteasome was Rpn10 (S5a). Deveraux et al. used proteasomes isolated from human red blood cells and observed that the 19S subunit Rpn10 bound ubiquitinated lysozyme conjugates [11]. However, this was clearly not the only ubiquitin receptor for the proteasome, as the deletion of Rpn10 in



facilitate binding of ubiquitinated proteins to the 19S (a). Ubl-UBA proteins, such as $\operatorname{Rad} 23$ and Dsk2, associate with the 26S through their Ubl domains that bind to either Rpn10 or Rpn13. The UBA domains bind the polyubiquitin chains (b)

saccharomyces cerevisiae had a modest phenotype and did not affect viability [12]. Subsequent studies in yeast have been instrumental in identifying other UBDs within other intrinsic components of the proteasome, namely, Rpt5, Rpn13, and Sem1 (Dss1) [4, 13, 14] (Fig. 1a; Table 1). While all these subunits can bind polyubiquitinated proteins, the relative contribution of each for facilitating proteasomal degradation is unclear. However, biochemical assays of ubiquitin binding and protein degradation show that the $19 \mathrm{~S}$ subunits, Rpn10 and Rpn13, are the high affinity ubiquitin receptors for the proteasome (Fig. 1a) [2-4, 15].

In addition to the intrinsic 19S ubiquitin receptors, the proteasome can bind polyubiquitinated substrates through proteins that transiently associate with the 19S (Fig. 1b; Table 1). Such proteins typically encode both ubiquitin like (Ubl) domains and UBDs. The UBDs bind the polyubiquitin chains, while the Ubl binds to the 19S high affinity ubiquitin receptors or other 19S subunits (e.g., Rpn1) [16]. Yeast $\operatorname{Rad} 23$ was one of the first Ubl-UBD proteins shown to bind polyubiquitinated proteins through its two UBA (ubiquitin-associated) domains and deliver these conjugates to the proteasome for degradation [16, 17]. However, the relative importance of Rad23 in recruiting polyubiquitinated proteins to the proteasome has subsequently been questioned, as although initial studies suggested that $\operatorname{Rad} 23$ and its human homologues (hHR23A and B) bind to polyubiquitinated proteins and the proteasome [18, 19], others found that $\operatorname{Rad} 23$ prevented protein degradation [20]. It is likely that these differences in $\operatorname{Rad} 23$ function are due to the protein concentration used in the in vitro assays 


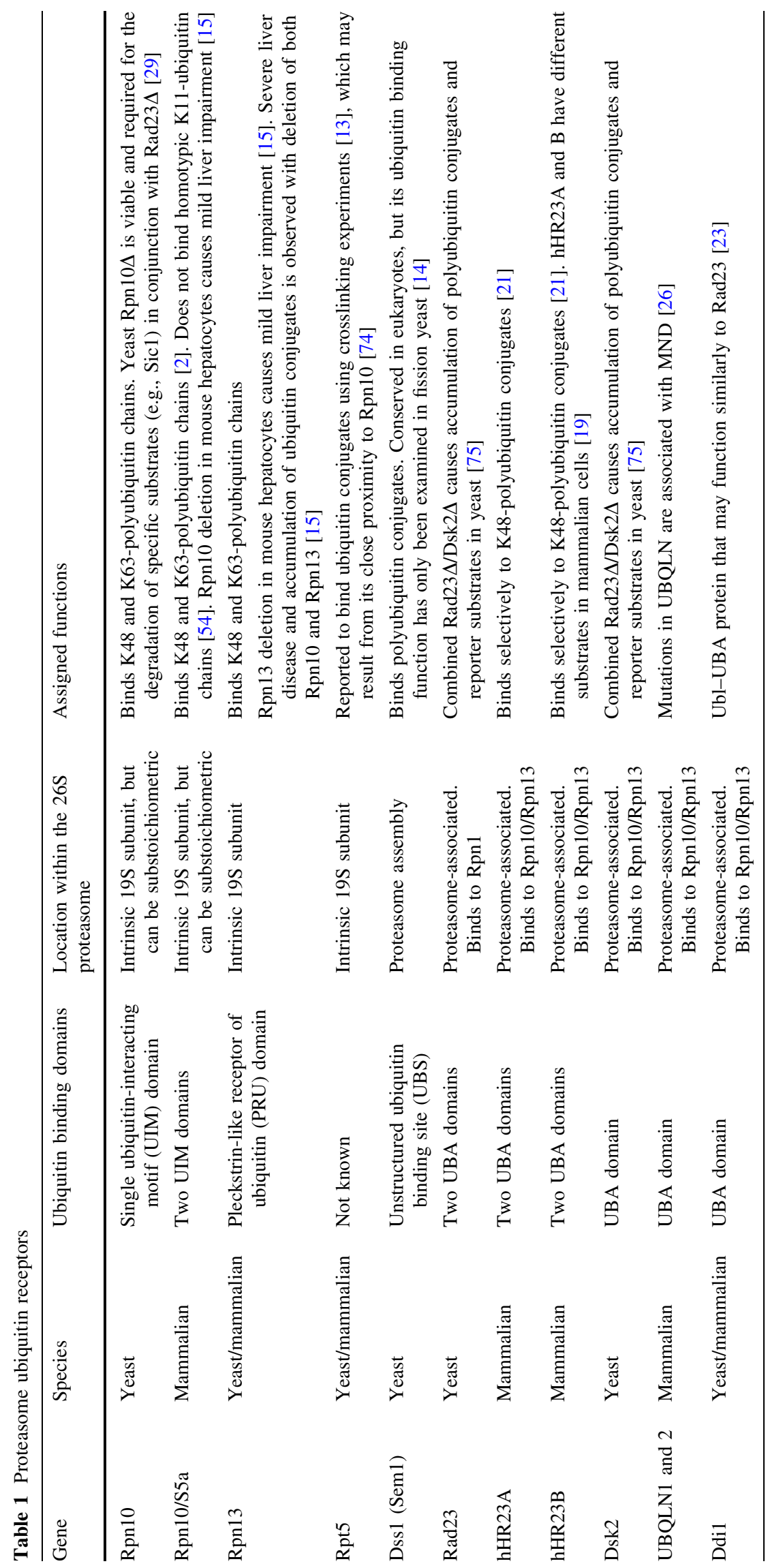


of proteasomal degradation. We found that when hHR23A and $\mathrm{B}$ are used at concentrations similar to those found in vivo, they stimulate the binding of K48-polyubiquitinated proteins to mammalian proteasomes [21].

Several other Ubl-UBA proteins have been identified, including Ddd1 [22, 23], Dsk2 [16, 24], and its human homologues [hPLIC1 and 2, also known as Ubiquilin (UBQLN1 and 2)] [25] (Table 1). The importance of these proteins is highlighted by the identification of human mutations causing neurological disease. Mutations in UBQLN2 cause an X-linked form of motor neurone disease [MND, or amyotrophic lateral sclerosis (ALS)] [26], while mutations in UBQLN1 are associated with MND as well as Alzheimer's disease [27, 28]. These mutations are thought to be pathogenic in part due to the accumulation of ubiquitinated protein aggregates and defects in proteasomal degradation [26].

With the relatively large number of known ubiquitin receptors that are either encoded within the 19S or can associate with the complex (Table 1), it is not surprising that the deletions of individual UBPs may not alter viability [29, 30]. However, it is likely that different proteasomeassociated UBPs bind distinct groups of polyubiquitinated proteins, allowing for selective and specific protein degradation. Indeed, Verma et al. show that while yeast deletion of Rad23 and Rpn10 does not affect overall protein degradation, it does prevent the degradation of specific proteasome substrates, such as Sic1 [29]. Similarly, in a mammalian system, Hamazaki et al. show that liverspecific deletion of either Rpn10 or Rpn13 results in modest impairment of function, but the deletion of both ubiquitin receptors causes severe liver injury and the accumulation of polyubiquitin conjugates [15].

\section{K48-polyubiquitin chains signal proteasome- mediated degradation}

K48-polyubiquitin chains are the most abundant linkage in cells [31, 32], and are thought to be the major signal for proteasome-mediated degradation [33, 34]. Quantitative mass spectrometry analyses of intracellular ubiquitin linkages support this notion, as K48-polyubiquitin linkages rapidly accumulate when cells are treated with the proteasome inhibitor MG132 [32]. Biochemical studies provided the first direct evidence for K48-polyubiquitin chains binding to the $26 \mathrm{~S}$ proteasome and stimulating protein degradation [33, 35]. Subsequently, Thrower et al. identified a chain of four or more K48-linked ubiquitins as the optimal chain length to stimulate protein degradation [35]. However, recent studies suggest that the number of K48-polyubiquitin chains may be more important than the chain length. In elegant proteasomal degradation assays and single molecule kinetic studies, Lu et al. demonstrate that multiple ubiquitinated lysines are more efficient than K48-polyubiquitin chains in stimulating proteasomal degradation [36]. They observed rapid degradation of the cell-cycle protein, cyclin B1, when modified with two diubiquitin K48-chains as compared with a single tetraubiquitin K48-chain [36]. It should be noted that human proteasomes used in these assays were salt washed to remove the DUB USP14 and associated proteins (including hHR23A and B). Whether Ubl-UBA proteins affect the degradation of ubiquitinated proteins modified with several K48-linked chains remains to be determined.

Biochemical assays confirm that K48-polyubiquitin conjugates initially bind to the proteasome through Rpn10 and Rpn13 (Fig. 2a). Proteasomes purified from yeast strains that lack UBDs in Rpn10 and Rpn13 (rpn10 1 UIM, rpn13KKD, or $\mathrm{rpn} 10 \Delta \mathrm{UIM} / \mathrm{rpn} 13 \mathrm{KKD}$ ) show reduced affinity for K48-polyubiquitin conjugates, with a fourfold decrease in affinity for the double mutant [2]. However, some K48-polyubiquitin conjugates still bind to proteasomes in these Rpn10/Rpn13 mutants, indicating the presence of lower affinity ubiquitin receptors for polyubiquitin chains [2].

Because K48-polyubiquitin chains are the major signal for proteasome-mediated degradation, it was possible that the intrinsic proteasome ubiquitin receptors selectively bind K48-linked chains. This is clearly not the case, as pure proteasomes bind K48- and K63-linked chains similarly, through the same high affinity Rpn10 and Rpn13 ubiquitin receptors [2, 37, 38]. However, Ubl-UBA proteins, such as Rad23, do show selectivity for K48-polyubiquitin chains. The second UBA domain of $\operatorname{Rad} 23$ has a higher affinity for K48-tetraubiquitin compared with K63-tetraubiquitin [39]. In addition, we found that full-length hHR23A and hHR23B bind only to K48-polyubiquitin conjugates when compared with K63-polyubiquitin chains [21]. Furthermore, full-length hHR23A and, particularly, hHR23B selectively stimulate K48-polyubiquitin conjugates binding to the $26 \mathrm{~S}$ proteasome [21]. Therefore, the $\operatorname{Rad} 23$ proteins do provide ubiquitin linkage specificity for delivering K48-polyubiquitinated proteins to the proteasome. Whether other proteasome-associated ubiquitin receptors share the same specificity for K48-linkages remains to be determined.

\section{K63-polyubiquitin chains bind to pure proteasomes but do not signal degradation in cells}

K63-polyubiquitin linkages are also highly abundant in cells [31, 32], but serve alternative functions to proteasome-mediated degradation, and are involved in intracellular signalling [40], DNA repair [41], and the targeting of proteins to the endosomal-lysosomal system 


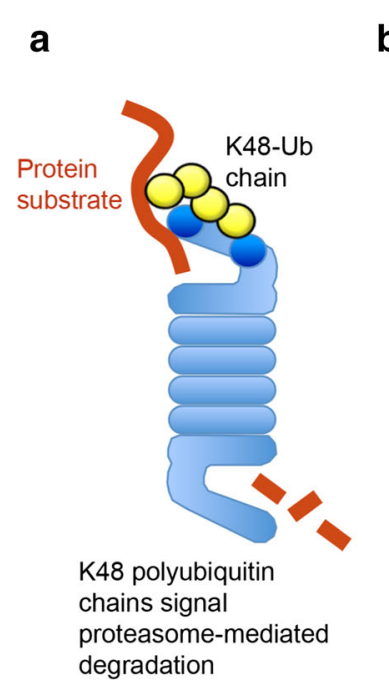

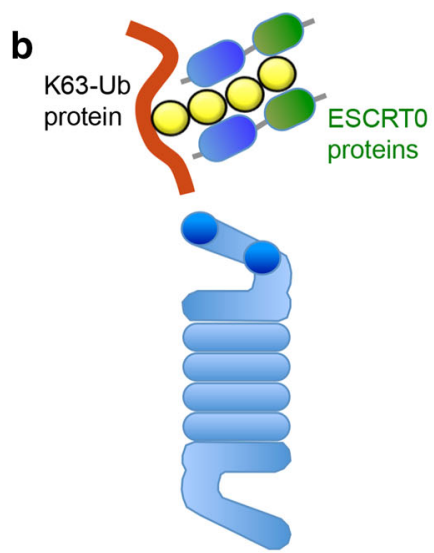

K63-polyubiquitinated proteins are prevented from binding to the $26 \mathrm{~S}$ in cells.
C

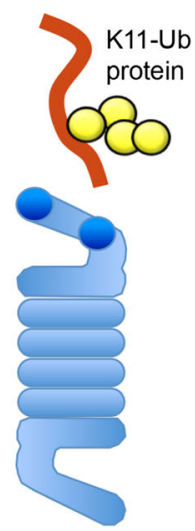

Homotypic K11polyubiquitinated proteins do not bind tightly to proteasome Ub receptors

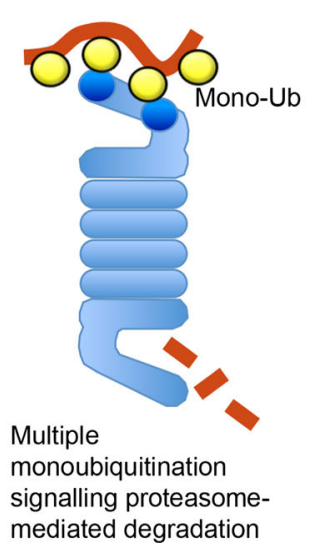

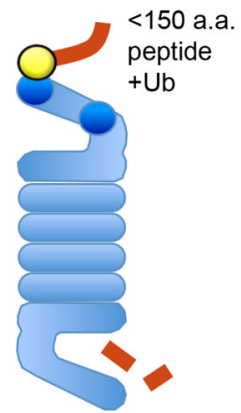
conjugated to short protein signals degradation
Single ubiquitin d

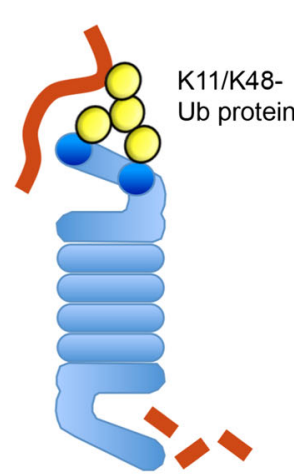

Heterotypic K11/K48polyubiquitin chains signal proteasomemediated degradation
Fig. 2 Recognition of different ubiquitin linkages by the $26 \mathrm{~S}$ proteasome. Schematic of the interaction of the $26 \mathrm{~S}$ proteasome with different types of ubiquitin linkages. The positions of Rpn13 and Rpn10 within the proteasome are highlighted (dark blue). K48polyubiquitinated proteins are the canonical signal for proteasomemediated degradation (a). K63-polyubiquitin chains are blocked from binding to the $26 \mathrm{~S}$ due to K63-selective ubiquitin binding proteins, which bind tightly to the K63-polyubiquitin chains and direct them to

[42]. One could, therefore, hypothesise that K63-polyubiquitinated proteins cannot bind to the proteasome. However, as previously mentioned, K63-polyubiquitin conjugates do not only bind pure $26 \mathrm{~S}$ proteasomes $[2,21]$ in vitro, but can also stimulate protein degradation at similar rates to K48-polyubiquitinated substrates [37, 38].

Three mechanisms could explain this discrepancy between the function of K63-polyubiquitinated chains in cells and their ability to signal proteasome-mediated degradation in vitro: (1) K63-polyubiquitin chains are rapidly disassembled in cells preventing their association with the $26 \mathrm{~S}$ proteasome. (2) K48-polyubiquitin chains are more efficiently delivered to the proteasome by proteins, such as hHR23B, and (3) K63-polyubiquitin chains are

alternative pathways (endosomal-lysosomal pathway) (b). Homotypic K11-polyubiquitin chains do not bind tightly to the $19 \mathrm{~S}$ proteasome receptors (c). Heterotypic K11/K48-polyubiquitinated proteins signal proteasome-mediated degradation of cell-cycle substrates (e.g., cyclin B1) (d). Multiple monoubiquitination can facilitate the degradation of some proteasome substrates (cyclin B1) (e). Short proteins (less than 150 amino acids) may be targeted for degradation by a single ubiquitin moiety (f). Ub ubiquitin

blocked from binding the $19 \mathrm{~S}$ by intracellular factors. Several groups have examined which of these mechanisms may account for preventing the degradation of K63polyubiquitin conjugates in cells.

Cooper et al. found that K63-polyubiquitin conjugates in cell lysates were rapidly disassembled compared with K48chains, and identified the BRISC (Brcc36 isopeptidase complex) as the DUB responsible for this observation [43]. Jacobson et al. also found that K63-polyubiquitin chains are more rapidly disassembled by the proteasome-associated DUBs [31]. The proteasome DUB Rpn11 (Poh1) and BRISC are members of the JAMM/MPN+ (Jab1/Mov34/ Mpr1 Pad1 N-terminal+) proteases, and it has been suggested that this group of DUBs shows a preference for 
K63-polyubiquitin chains [43]. However, it is unlikely that rapid disassembly of K63-conjugates provides the major mechanism for protecting K63-polyubiquitinated proteins, as the binding of polyubiquitin chains to the high affinity proteasome ubiquitin receptors precedes DUB activation. Furthermore, by measuring conjugate binding at $4{ }^{\circ} \mathrm{C}$, we showed that both $\mathrm{K} 48$ - and K63-polyubiquitin conjugates bind similarly, and we observed no difference in this binding when DUB inhibitors were added [21].

To identify factors in cells that may prevent K63polyubiquitin chains from binding to the proteasome, we used a biochemical and mass spectrometry approach. Several proteins that bound strongly to K63-polyubiquitin conjugates were identified, including the ESCRT0 proteins [21]. This heterodimeric complex recruits polyubiquitinated proteins to the endosomal-lysosomal pathway (reviewed in [44]), but is also present within the cytosol [21]. We found that ESCRT0 not only selectively bound to K63-polyubiquitin chains, but also prevented these conjugates from binding to pure proteasomes. Thus, K48selective Ubl-UBA proteins (e.g., hHR23B) stimulate K48-polyubiquitin conjugates binding to the proteasome, and K63-polyubiquitin chains are protected from proteasomal degradation by K63-linkage-specific UBPs, such as ESCRT0 (Fig. 2b) [21].

\section{Homotypic K11-polyubiquitin chains do not bind to the proteasome}

K11-linked polyubiquitin chains are the third most abundant linkages in cells [32], and have a unique structure compared with K48, K63, or linear-linked chains [45]. The most well characterized role for K11-linkages is in the cell cycle. In mitosis, the anaphase promoting complex/cyclosome (APC/C) E3 ligase catalyzes polyubiquitin chain formation on cell-cycle proteins (e.g., cyclin B1 and securin) signalling the degradation of these mitotic regulators [46, 47]. K11-chains have also been shown to accumulate in cells released from mitotic arrest, when the $\mathrm{APC} / \mathrm{C}$ is most active [48]. In vitro studies have identified that several E2 enzymes are required for the ubiquitination of APC/C substrates. Either UbcH10 or UbcH5 is required to initiate ubiquitination of cyclin $\mathrm{B} 1$, and the polyubiquitin chain formed is then extended by the K11-specific E2 enzyme Ube2S [48, 49]. It was, therefore, initially thought that K11-chains were required for the degradation of cellcycle checkpoint proteins. Interestingly though, knockdown of Ube $2 \mathrm{~S}$ by siRNA-depletion in HeLa cells only delayed exit from mitosis when drug-induced perturbations (e.g., monastrol) of the cell cycle were used, and Ube2S was not required for normal mitosis [49]. In addition, Ube $2 \mathrm{~S}$ is not necessary for degradation of ubiquitinated cyclin B1, as multiple monoubiquitination of cyclin B1 was sufficient to initiate its proteasome-mediated degradation [36, 50].

In contrast to the presumed proteasome-mediated degradative role of K11-linkages in the cell cycle, K11polyubiquitination has been observed in other cellular processes, including ER-associated degradation (ERAD) [32], the hypoxia response [51], mitophagy [52], and even in stabilizing proteins, such as $\beta$-catenin [53].

Using pure $26 \mathrm{~S}$ proteasomes and homotypic K11polyubiquitin conjugates, we examined whether these chains facilitated proteasomal degradation. Surprisingly, we found that homotypic K11-chains did not bind to pure proteasomes or to the proteasome-associated ubiquitin receptors (Fig. 2c) [54]. In addition, homotypic K11polyubiquitin chains did not facilitate the rapid degradation of cyclin B1. The finding that homotypic K11-polyubiquitination does not signal proteasomal degradation has been subsequently observed by Martinez-Fonts et al, who used a fluorescent-based proteasomal degradation assay of K11-polyubiquitinated GFP [55].

We also examined the binding of homotypic K11polyubiquitin chains to the $19 \mathrm{~S}$ ubiquitin receptors and Ubl-UBA proteins. Consistent with K11-polyubiquitin chains being a weak signal for proteasomal degradation, we observed no significant binding of homotypic K11-chains to Rpn10, hHR23A, hHR23B, or UBQLN1 [54]. Other groups have examined the direct binding of UBDs within proteasome-associated ubiquitin receptors and K11-chains. Castaneda et al. show that the UBA2 domain of hHR23A binds K48 dimers more strongly than K11-ubiquitin dimers [45]. It appears that a single molecule of UBA cannot interact with both hydrophobic regions of the K11-ubiquitin dimers at the same time. This is not the case for K48 ubiquitin dimers, which can associate with a single UBA moiety [45]. The compact K11-ubiquitin dimer formation and orientation of the two ubiquitin molecules may prevent the I44 hydrophobic region being available to the proteasomal ubiquitin receptors, hence explaining the weak binding of these chains in comparison with K48-ubiquitin linkages [54].

\section{Heterotypic K11/K48-polyubiquitin chains signal proteasome-mediated degradation}

It is likely that the diverse fates of K11-polyubiquitinated proteins relates to the homotypic or heterotypic conformation of the chain. Recent studies show that the APC/C forms heterotypic K11/K48-chains on cell-cycle substrates (Nek2A and cyclin A), rather than homotypic K11-chains [56]. Mayer et al. observed that these heterotypic chains were more efficient at stimulating the degradation of the 
cell-cycle substrates compared with homotypic K48polyubiquitin chains (Fig. 2d) [56]. While we also found that heterotypic K11/K48-polyubiquitin chains bind to the proteasome and signal degradation, they were not as efficient as homotypic K48-polyubiquitin chains [54]. The reason for this discrepancy may relate to the experimental conditions and our use of recombinant $\mathrm{APC} / \mathrm{C}$ rather than APC/C immunoprecipitated from cell extracts.

The ability of the proteasome to differentially degrade homotypic and heterotypic K11-polyubiquitin conjugates may also relate to disassembly of the chains by the proteasome-associated DUBs. We observed that K11/K48heterotypic chains were disassembled slowly compared with the K11-homotypic chains [54]. Mansour et al. also found that K11-polyubiquitin chains were rapidly disassembled by the proteasome-associated DUBs compared with K48-chains. How the heterotypic chains bind and interact with the proteasome-associated DUBs is unclear. Furthermore, whether K11-heterotypic chains containing other lysine linkages can be disassembled by these DUBs, or stimulate proteasomal degradation is not known. However, it is noteworthy that complex polyubiquitin chains, containing forked chains of mixed linkages have been reported to block proteasomal degradation [57].

\section{Monoubiquitination as a signal for proteasome- mediated degradation}

Monoubiquitination is typically involved in subcellular localization of proteins [58] or signalling the internalization of plasma membrane receptors, rather than facilitating degradation [59]. However, the requirement for a polyubiquitin chain of four or more ubiquitin moieties has been challenged by several recent studies. Dimova et al. showed that multiple monoubiquitination of cyclin B1 is an efficient signal for proteasomal degradation in Xenopus extracts (Fig. 2e) [50]. Lu et al. confirm that multiple monoubiquitination of cyclin B1 is sufficient to stimulate degradation by human proteasomes using single molecule kinetic studies [36]. However, multiple monoubiquitination of other cell-cycle substrates (geminin and securin) did not facilitate rapid proteasomal degradation [36]. The distribution and conformation of the multi-monoubiquitinated protein may be important for binding to the proteasome ubiquitin receptors. In addition, the position of unfolded regions within the ubiquitinated substrate will contribute to the rate of proteasomal degradation [60]. Indeed, Shabek et al. show that a single ubiquitin fused to an approximately 150 amino acid polypeptide (repeat HA tag) was sufficient to signal proteasomal degradation (Fig. 2f) [61]. However, ubiquitin fused to polypeptides longer than 150 amino acids were degraded slowly.

\section{What is the role of other ubiquitin linkages in signalling proteasomal degradation?}

Whether the other ubiquitin linkages that occur in cells can mediate proteasomal degradation is largely unknown. Linear chains are structurally similar to K63-polyubiquitin chains, and appear confined to $\mathrm{NF}-\kappa \mathrm{B}$ signalling [62]. UBPs, such as NEMO (NF- $\kappa \mathrm{B}$ essential modulator), selectively bind linear chains [63], and may prevent them binding to the proteasome, similarly to ESCRT0 and K63polyubiquitin chains [21].

While K6, K27, K29, and K33 ubiquitin linkages are all detected at low levels in cells, they do all increase to varying extents when cells are treated with proteasome inhibitors [32]. This suggests that they may be involved in proteasome-mediated degradation, but not necessarily in homotypic conformations. However, the role of these ubiquitin linkages in cells remains unclear. K6 and K27 linkages are associated with DNA replication repair [64, 65], while K29 and K33 are hydrolyzed by the DUB involved in Wnt signalling (TRABID) [66]. Structural analyses of dimeric forms of these atypical ubiquitin linkages reveal distinct conformations [67-69], suggesting that they can form unique ubiquitin signals. Whether they have a role in proteasome-mediated degradation remains to be determined.

\section{Future perspectives}

Advances in biochemical techniques and single molecule kinetics will offer further mechanistic insights into ubiquitin recognition by the $26 \mathrm{~S}$ proteasome. Particularly, the ability to form different polyubiquitin linked chains of defined length will help to elucidate the role of atypical ubiquitin linkages in proteasome-mediated degradation. However, determining the type and number of ubiquitin chains that occur on individual protein substrates in cells remains a challenge. Post-translational modifications of ubiquitin and its receptors add a further level of complexity to the system. For example, phosphorylation of ubiquitin at serine 65 (S65) by PTEN-induced putative kinase 1 (PINK1) is required for the clearance of damaged mitochondria [70-72]. While S65 phosphorylated ubiquitin can be incorporated into different polyubiquitin chains [73], the role of the proteasome in degrading such conjugates is not known. It will be of interest to examine how S65 phosphorylation and other modifications of ubiquitin are involved in proteasome-mediated degradation.

Acknowledgments GLG and JAN wrote the manuscript. We would like to thank members of the Nathan laboratory for their helpful discussions and comments. JAN is supported by a Wellcome Trust 
Senior Clinical Research Fellowship (102770/Z/13/Z). The Cambridge Institute for Medical Research is in receipt of a Wellcome Trust Strategic Award (100140).

Open Access This article is distributed under the terms of the Creative Commons Attribution 4.0 International License (http:// creativecommons.org/licenses/by/4.0/), which permits unrestricted use, distribution, and reproduction in any medium, provided you give appropriate credit to the original author(s) and the source, provide a link to the Creative Commons license, and indicate if changes were made.

\section{References}

1. Husnjak K, Dikic I (2012) Ubiquitin-binding proteins: decoders of ubiquitin-mediated cellular functions. Annu Rev Biochem 81:291-322

2. Peth A, Uchiki T, Goldberg AL (2010) ATP-dependent steps in the binding of ubiquitin conjugates to the $26 \mathrm{~S}$ proteasome that commit to degradation. Mol Cell 40:671-681

3. Schreiner P, Chen X, Husnjak K, Randles L, Zhang N, Elsasser S, Finley D, Dikic I, Walters KJ, Groll M (2008) Ubiquitin docking at the proteasome through a novel Pleckstrin-homology domain interaction. Nature 453:548-552

4. Husnjak K, Elsasser S, Zhang N, Chen X, Randles L, Shi Y, Hofmann K, Walters KJ, Finley D, Dikic I (2008) Proteasome subunit Rpn13 is a novel ubiquitin receptor. Nature 453:481-488

5. Finley D (2009) Recognition and processing of ubiquitin-protein conjugates by the proteasome. Annu Rev Biochem 78:477-513

6. Smith DM, Chang SC, Park S, Finley D, Cheng Y, Goldberg AL (2007) Docking of the proteasomal ATPases' carboxyl termini in the 20S proteasome's alpha ring opens the gate for substrate entry. Mol Cell 27:731-744

7. Peth A, Besche HC, Goldberg AL (2009) Ubiquitinated proteins activate the proteasome by binding to Usp14/Ubp6, which causes 20S gate opening. Mol Cell 36:794-804

8. Smith DM, Fraga H, Reis C, Kafri G, Goldberg AL (2011) ATP binds to proteasomal ATPases in pairs with distinct functional effects, implying an ordered reaction cycle. Cell 144:526-538

9. Hurley JH, Lee S, Prag G (2006) Ubiquitin-binding domains. Biochem J 399:361-372

10. Dikic I, Wakatsuki S, Walters KJ (2009) Ubiquitin-binding domains-from structures to functions. Nat Rev Mol Cell Biol 10:659-671

11. Deveraux Q, Ustrell V, Pickart C, Rechsteiner M (1994) A 26 S protease subunit that binds ubiquitin conjugates. J Biol Chem 269:7059-7061

12. van Nocker S, Sadis S, Rubin DM, Glickman M, Fu H, Coux O, Wefes I, Finley D, Vierstra RD (1996) The multiubiquitin-chainbinding protein Mcb1 is a component of the $26 \mathrm{~S}$ proteasome in Saccharomyces cerevisiae and plays a nonessential, substratespecific role in protein turnover. Mol Cell Biol 16:6020-6028

13. Lam YA, Lawson TG, Velayutham M, Zweier JL, Pickart CM (2002) A proteasomal ATPase subunit recognizes the polyubiquitin degradation signal. Nature 416:763-767

14. Paraskevopoulos K, Kriegenburg F, Tatham MH, Rosner HI, Medina B, Larsen IB, Brandstrup R, Hardwick KG, Hay RT, Kragelund BB, Hartmann-Petersen R, Gordon C (2014) Dss1 is a 26S proteasome ubiquitin receptor. Mol Cell 56:453-461

15. Hamazaki J, Hirayama S, Murata S (2015) Redundant Roles of Rpn10 and Rpn13 in recognition of ubiquitinated proteins and cellular homeostasis. PLoS Genet 11:e1005401
16. Elsasser S, Gali RR, Schwickart M, Larsen CN, Leggett DS, Muller B, Feng MT, Tubing F, Dittmar GA, Finley D (2002) Proteasome subunit Rpn1 binds ubiquitin-like protein domains. Nat Cell Biol 4:725-730

17. Schauber C, Chen L, Tongaonkar P, Vega I, Lambertson D, Potts W, Madura K (1998) Rad23 links DNA repair to the ubiquitin/ proteasome pathway. Nature 391:715-718

18. Chen L, Madura K (2002) Rad23 promotes the targeting of proteolytic substrates to the proteasome. Mol Cell Biol 22:4902-4913

19. Chen L, Madura K (2006) Evidence for distinct functions for human DNA repair factors hHR23A and hHR23B. FEBS Lett 580:3401-3408

20. Raasi S, Pickart CM (2003) Rad23 ubiquitin-associated domains (UBA) inhibit $26 \mathrm{~S}$ proteasome-catalyzed proteolysis by sequestering lysine 48 -linked polyubiquitin chains. J Biol Chem 278:8951-8959

21. Nathan JA, Kim HT, Ting L, Gygi SP, Goldberg AL (2013) Why do cellular proteins linked to K63-polyubiquitin chains not associate with proteasomes? EMBO J 32:552-565

22. Diaz-Martinez LA, Kang Y, Walters KJ, Clarke DJ (2006) Yeast UBL-UBA proteins have partially redundant functions in cell cycle control. Cell Div 1:28

23. Nowicka U, Zhang D, Walker O, Krutauz D, Castaneda CA, Chaturvedi A, Chen TY, Reis N, Glickman MH, Fushman D (2015) DNA-damage-inducible 1 protein (Ddi1) contains an uncharacteristic ubiquitin-like domain that binds ubiquitin. Structure. 23:542-557

24. Medicherla B, Kostova Z, Schaefer A, Wolf DH (2004) A genomic screen identifies Dsk2p and Rad23p as essential components of ER-associated degradation. EMBO Rep 5:692-697

25. Kleijnen MF, Shih AH, Zhou P, Kumar S, Soccio RE, Kedersha NL, Gill G, Howley PM (2000) The hPLIC proteins may provide a link between the ubiquitination machinery and the proteasome. Mol Cell 6:409-419

26. Deng HX, Chen W, Hong ST, Boycott KM, Gorrie GH, Siddique N, Yang Y, Fecto F, Shi Y, Zhai H, Jiang H, Hirano M, Rampersaud E, Jansen GH, Donkervoort S, Bigio EH, Brooks BR, Ajroud K, Sufit RL, Haines JL, Mugnaini E, Pericak-Vance MA, Siddique $\mathrm{T}$ (2011) Mutations in UBQLN2 cause dominant $\mathrm{X}$-linked juvenile and adult-onset ALS and ALS/dementia. Nature 477:211-215

27. Kim SH, Shi Y, Hanson KA, Williams LM, Sakasai R, Bowler MJ, Tibbetts RS (2009) Potentiation of amyotrophic lateral sclerosis (ALS)-associated TDP-43 aggregation by the proteasome-targeting factor, ubiquilin 1. J Biol Chem 284:8083-8092

28. Viswanathan J, Haapasalo A, Bottcher C, Miettinen R, Kurkinen KM, Lu A, Thomas A, Maynard CJ, Romano D, Hyman BT, Berezovska O, Bertram L, Soininen H, Dantuma NP, Tanzi, RE, Hiltunen M (2011) Alzheimer's disease-associated ubiquilin-1 regulates presenilin-1 accumulation and aggresome formation. Traffic (Copenhagen, Denmark) 12:330-348

29. Verma R, Oania R, Graumann J, Deshaies RJ (2004) Multiubiquitin chain receptors define a layer of substrate selectivity in the ubiquitin-proteasome system. Cell 118:99-110

30. Elsasser S, Chandler-Militello D, Muller B, Hanna J, Finley D (2004) Rad23 and Rpn10 serve as alternative ubiquitin receptors for the proteasome. J Biol Chem 279:26817-26822

31. Jacobson AD, Zhang NY, Xu P, Han KJ, Noone S, Peng J, Liu $\mathrm{CW}$ (2009) The lysine 48 and lysine 63 ubiquitin conjugates are processed differently by the $26 \mathrm{~s}$ proteasome. J Biol Chem 284:35485-35494

32. Xu P, Duong DM, Seyfried NT, Cheng D, Xie Y, Robert J, Rush J, Hochstrasser M, Finley D, Peng J (2009) Quantitative proteomics reveals the function of unconventional ubiquitin chains in proteasomal degradation. Cell 137:133-145 
33. Chau V, Tobias JW, Bachmair A, Marriott D, Ecker DJ, Gonda DK, Varshavsky A (1989) A multiubiquitin chain is confined to specific lysine in a targeted short-lived protein. Science 243:1576-1583

34. Finley D, Sadis S, Monia BP, Boucher P, Ecker DJ, Crooke ST, Chau V (1994) Inhibition of proteolysis and cell cycle progression in a multiubiquitination-deficient yeast mutant. Mol Cell Biol 14:5501-5509

35. Thrower JS, Hoffman L, Rechsteiner M, Pickart CM (2000) Recognition of the polyubiquitin proteolytic signal. EMBO $\mathrm{J}$ 19:94-102

36. Lu Y, Lee BH, King RW, Finley D, Kirschner MW (2015) Substrate degradation by the proteasome: a single-molecule kinetic analysis. Science 348:1250834

37. Kim HT, Kim KP, Lledias F, Kisselev AF, Scaglione KM, Skowyra D, Gygi SP, Goldberg AL (2007) Certain pairs of ubiquitin-conjugating enzymes (E2s) and ubiquitin-protein ligases (E3s) synthesize nondegradable forked ubiquitin chains containing all possible isopeptide linkages. J Biol Chem 282:17375-17386

38. Saeki Y, Kudo T, Sone T, Kikuchi Y, Yokosawa H, Toh-e A, Tanaka K (2009) Lysine 63-linked polyubiquitin chain may serve as a targeting signal for the 26S proteasome. EMBO J 28:359-371

39. Raasi S, Orlov I, Fleming KG, Pickart CM (2004) Binding of polyubiquitin chains to ubiquitin-associated (UBA) domains of HHR23A. J Mol Biol 341:1367-1379

40. Deng L, Wang C, Spencer E, Yang L, Braun A, You J, Slaughter C, Pickart C, Chen ZJ (2000) Activation of the IkappaB kinase complex by TRAF6 requires a dimeric ubiquitin-conjugating enzyme complex and a unique polyubiquitin chain. Cell 103:351-361

41. Hofmann RM, Pickart CM (1999) Noncanonical MMS2-encoded ubiquitin-conjugating enzyme functions in assembly of novel polyubiquitin chains for DNA repair. Cell 96:645-653

42. Duncan LM, Piper S, Dodd RB, Saville MK, Sanderson CM, Luzio JP, Lehner PJ (2006) Lysine-63-linked ubiquitination is required for endolysosomal degradation of class I molecules. EMBO J 25:1635-1645

43. Cooper EM, Cutcliffe C, Kristiansen TZ, Pandey A, Pickart CM, Cohen RE (2009) K63-specific deubiquitination by two JAMM/ MPN+ complexes: BRISC-associated Brcc36 and proteasomal Poh1. EMBO J 28:621-631

44. Shields SB, Piper RC (2011) How ubiquitin functions with Escrts. Traffic, Copenhagen

45. Castaneda CA, Kashyap TR, Nakasone MA, Krueger S, Fushman D (2013) Unique structural, dynamical, and functional properties of k11-linked polyubiquitin chains. Structure 21:1168-1181

46. Pines J (2011) Cubism and the cell cycle: the many faces of the APC/C. Nat Rev Mol Cell Biol 12:427-438

47. Min M, Lindon C (2012) Substrate targeting by the ubiquitinproteasome system in mitosis. Semin Cell Dev Biol 23:482-491

48. Jin L, Williamson A, Banerjee S, Philipp I, Rape M (2008) Mechanism of ubiquitin-chain formation by the human anaphasepromoting complex. Cell 133:653-665

49. Garnett MJ, Mansfeld J, Godwin C, Matsusaka T, Wu J, Russell P, Pines J, Venkitaraman AR (2009) UBE2S elongates ubiquitin chains on APC/C substrates to promote mitotic exit. Nat Cell Biol 11:1363-1369

50. Dimova NV, Hathaway NA, Lee BH, Kirkpatrick DS, Berkowitz ML, Gygi SP, Finley D, King RW (2012) APC/C-mediated multiple monoubiquitylation provides an alternative degradation signal for cyclin B1. Nat Cell Biol 14:168-176

51. Bremm A, Moniz S, Mader J, Rocha S, Komander D (2014) Cezanne (OTUD7B) regulates HIF-1alpha homeostasis in a proteasome-independent manner. EMBO Rep 15:1268-1277

52. Cunningham CN, Baughman JM, Phu L, Tea JS, Yu C, Coons M, Kirkpatrick DS, Bingol B, Corn JE (2015) USP30 and parkin homeostatically regulate atypical ubiquitin chains on mitochondria. Nat Cell Biol 17:160-169

53. Dao KH, Rotelli MD, Petersen CL, Kaech S, Nelson WD, Yates JE, Hanlon Newell AE, Olson SB, Druker BJ, Bagby GC (2012) FANCL ubiquitinates beta-catenin and enhances its nuclear function. Blood 120:323-334

54. Grice GL, Lobb IT, Weekes MP, Gygi SP, Antrobus R, Nathan JA (2015) The proteasome distinguishes between heterotypic and homotypic lysine-11-linked polyubiquitin chains. Cell Rep. 12:545-553

55. Martinez-Fonts K, Matouschek A (2016) A rapid and versatile method to generate proteins with defined ubiquitin chains. Biochemistry 55(12):1898-1908. doi:10.1021/acs.biochem.5b01310

56. Meyer HJ, Rape M (2014) Enhanced protein degradation by branched ubiquitin chains. Cell 157:910-921

57. Kim HT, Kim KP, Uchiki T, Gygi SP, Goldberg AL (2009) S5a promotes protein degradation by blocking synthesis of nondegradable forked ubiquitin chains. EMBO J 28:1867-1877

58. van der Horst A, de Vries-Smits AM, Brenkman AB, van Triest $\mathrm{MH}$, van den Broek N, Colland F, Maurice MM, Burgering BM (2006) FOXO4 transcriptional activity is regulated by monoubiquitination and USP7/HAUSP. Nat Cell Biol 8:1064-1073

59. Hicke L (2001) Protein regulation by monoubiquitin. Nat Rev Mol Cell Biol 2:195-201

60. Prakash S, Tian L, Ratliff KS, Lehotzky RE, Matouschek A (2004) An unstructured initiation site is required for efficient proteasome-mediated degradation. Nat Struct Mol Biol 11:830-837

61. Shabek N, Herman-Bachinsky Y, Buchsbaum S, Lewinson O, Haj-Yahya M, Hejjaoui M, Lashuel HA, Sommer T, Brik A, Ciechanover A (2012) The size of the proteasomal substrate determines whether its degradation will be mediated by mono- or polyubiquitylation. Mol Cell 48:87-97

62. Tokunaga F, Sakata S, Saeki Y, Satomi Y, Kirisako T, Kamei K, Nakagawa T, Kato M, Murata S, Yamaoka S, Yamamoto M, Akira S, Takao T, Tanaka K, Iwai K (2009) Involvement of linear polyubiquitylation of NEMO in NF-kappaB activation. Nat Cell Biol 11:123-132

63. Kensche T, Tokunaga F, Ikeda F, Goto E, Iwai K, Dikic I (2012) Analysis of nuclear factor-kappaB (NF-kappaB) essential modulator (NEMO) binding to linear and lysine-linked ubiquitin chains and its role in the activation of NF-kappaB. J Biol Chem 287:23626-23634

64. Morris JR, Solomon E (2004) BRCA1: BARD1 induces the formation of conjugated ubiquitin structures, dependent on K6 of ubiquitin, in cells during DNA replication and repair. Hum Mol Genet 13:807-817

65. Gatti M, Pinato S, Maiolica A, Rocchio F, Prato MG, Aebersold R, Penengo L (2015) RNF168 promotes noncanonical K27 ubiquitination to signal DNA damage. Cell Rep. 10:226-238

66. Licchesi JD, Mieszczanek J, Mevissen TE, Rutherford TJ, Akutsu M, Virdee S, El Oualid F, Chin JW, Ovaa H, Bienz M, Komander D (2012) An ankyrin-repeat ubiquitin-binding domain determines TRABID's specificity for atypical ubiquitin chains. Nat Struct Mol Biol 19:62-71

67. Castaneda CA, Dixon EK, Walker O, Chaturvedi A, Nakasone MA, Curtis JE, Reed MR, Krueger S, Cropp TA, Fushman D (2016) Linkage via K27 bestows ubiquitin chains with unique properties among polyubiquitins. Structure. 24:423-436

68. Kristariyanto YA, Abdul Rehman SA, Campbell DG, Morrice NA, Johnson C, Toth R, Kulathu Y (2015) K29-selective ubiquitin binding domain reveals structural basis of specificity and heterotypic nature of k29 polyubiquitin. Mol Cell 58:83-94

69. Michel MA, Elliott PR, Swatek KN, Simicek M, Pruneda JN, Wagstaff JL, Freund SM, Komander D (2015) Assembly and 
specific recognition of $\mathrm{k} 29$ - and $\mathrm{k} 33$-linked polyubiquitin. Mol Cell 58:95-109

70. Kane LA, Lazarou M, Fogel AI, Li Y, Yamano K, Sarraf SA, Banerjee S, Youle RJ (2014) PINK1 phosphorylates ubiquitin to activate Parkin E3 ubiquitin ligase activity. J Cell Biol 205:143-153

71. Kazlauskaite A, Kondapalli C, Gourlay R, Campbell DG, Ritorto MS, Hofmann K, Alessi DR, Knebel A, Trost M, Muqit MM (2014) Parkin is activated by PINK1-dependent phosphorylation of ubiquitin at Ser65. Biochem J 460:127-139

72. Koyano F, Okatsu K, Kosako H, Tamura Y, Go E, Kimura M, Kimura Y, Tsuchiya H, Yoshihara H, Hirokawa T, Endo T, Fon EA, Trempe JF, Saeki Y, Tanaka K, Matsuda N (2014) Ubiquitin is phosphorylated by PINK1 to activate Parkin. Nature 510:162-166
73. Wauer T, Swatek KN, Wagstaff JL, Gladkova C, Pruneda JN, Michel MA, Gersch M, Johnson CM, Freund SM, Komander D (2015) Ubiquitin Ser65 phosphorylation affects ubiquitin structure, chain assembly and hydrolysis. EMBO J 34:307-325

74. Sakata E, Bohn S, Mihalache O, Kiss P, Beck F, Nagy I, Nickell S, Tanaka K, Saeki Y, Förster F, Baumeister W (2012) Localization of the proteasomal ubiquitin receptors Rpn10 and Rpn13 by electron cryomicroscopy. Proc Natl Acad Sci USA 109:1479-1484

75. Rao H, Sastry A (2002) Recognition of specific ubiquitin conjugates is important for the proteolytic functions of the ubiquitinassociated domain proteins Dsk2 and Rad23. J Biol Chem 277:11691-11695 